\title{
La fachada ventilada y su posible adaptación en ciudades de la región de Cuyo, Argentina
}

\author{
The Ventilated Façade and its possible adaptation in cities of the
} Cuyo region, Argentina

\section{Julieta Balter}

Instituto de Ambiente, Hábitat y Energía. Mendoza (Argentina) Consejo Nacional de Investigaciones Científicas y Técnicas. (Argentina)

\section{Virginia Miranda-Gassull}

Universidad Nacional de Cuyo. Mendoza (Argentina)

Consejo Nacional de Investigaciones Científicas y Técnicas. (Argentina) Instituto de Cartografía, Investigaciones y Formación

para el Ordenamiento Territorial (CIFOT)

\section{Carlos Discoli}

Universidad Nacional de La Plata. La Plata (Argentina)

Consejo Nacional de Investigaciones Científicas y Técnicas (Argentina)

Instituto de Investigaciones y Políticas del Ambiente Construido (IIPAC)

Balter, J., Miranda-Gassull, V., \& Discoli, C. (2021). La fachada ventilada y su posible adaptación en ciudades de la región de Cuyo, Argentina. Revista de Arquitectura (Bogotá), 23(2), 94-105. https://doi org/l0.14718/RevArg.2021.3338

\section{Julieta Balte}

Arquitecta. Universidad de Mendoza (Argentina)

Doctora en Arquitectura. Universidad Nacional de La Plata (Argentina). Investigadora Asistente CONICET.

https://scholar.google.es/scholar?hl=es\&as_sdt=0\%2C5\&q=julieta + balter\&btnG =

(Di) https://orcid.org/0000-0002-7785-8465

(10) jbalter@mendoza-conicet.gob.ar

Virginia Miranda-Gassull

Arquitecta. Universidad de Mendoza (Argentina)

Doctora Ordenamiento Territorial y Desarrollo Sostenible. Universidad Nacional de Cuyo (Argentina).

Investigadora Asistente, CONICET.

https://scholar.google.com/citations?user=GJ I hilsAAAAJ\&hl=es

(D)https://orcid.org/0000-000I-9382-5906

(10arq.vmiranda@gmail.com

Carlos Discoli

Ingeniero Mecánico. Universidad Tecnológica Nacional de La Plata (Argentina)

Doctor en Ciencias, Energías Renovables y Ambiente. Universidad Nacional de Salta (Argentina).

Investigador Principal CONICET.

-https://scholar.google.com/scholar?hl=es\&as_sdt=0\%2C5\&q=dis coli\&btnG =

iDhttps://orcid.org/0000-0002-6944-5492

(a)discoli@rocketmail.com

\section{Resumen}

Se busca identificar las posibilidades de insertar el sistema de envolvente de fachadas ventiladas en el contexto urbano-edilicio de la región de Cuyo, Argentina. A tal fin se consideran dos áreas de análisis: primero, la ciudad de Barcelona, España, donde se contrastan resultados del monitoreo de casos en relación con las normativas de edificación. Segundo, la ciudad de Mendoza, Argentina, donde se abarcan dos dimensiones: por un lado, si existe promoción para la implementación de nuevas tecnologías desde los dispositivos jurídicos y normativos; por otro, el análisis económico de los materiales necesarios para adaptar la tecnología local disponible al sistema de envolvente en estudio, así como las reducciones en los consumos energéticos para climatización que el sistema implica. Los resultados del trabajo advierten limitaciones instrumentales debido a los complejos requerimientos del código de edificación local para la aprobación y la implementación de nuevas tecnologías. Respecto a los costos para adaptar la tecnología local al sistema en estudio, estos resultan diez veces menores que los importados disponibles. Así mismo, el análisis edilicio interior por simulación dinámica mostró reducciones en los consumos energéticos para climatización del orden del $20 \%$. Los resultados advierten potenciales oportunidades para el desarrollo local de soluciones integrales, mediante tecnologías tendientes a la eficiencia energética, que incluyan a todos los sectores de la ciudad y su diversidad constructiva.

Palabras clave: diversidad constructiva; eficiencia energética; normativas de edificación; tecnologías de envolvente

\begin{abstract}
This paper aims to identify the possibilities of inserting the Ventilated Façade envelope system in the urban-building context of the Cuyo region, Argentina. To this end, two areas of analysis are considered: first, the city of Barcelona, Spain, where the results of case monitoring concerning building regulations are contrasted. Second, the city of Mendoza, Argentina, where two dimensions are covered: on the one hand, if there is a promotion for the implementation of new technologies from the legal and regulatory devices; and on the other hand, the economic analysis of the materials necessary to adapt the local technology available to the envelope system under study, as well as the reductions in energy consumption for air conditioning that the system implies. The results of the work point out instrumental limitations, due to the complex requirements of the local building code for the approval and implementation of new technologies. Regarding the costs to adapt the local technology to the system under study, these are ten times lower than the available imported ones. Likewise, the internal building analysis by dynamic simulation showed reductions in energy consumption for air conditioning in the order of $20 \%$. The results reveal potential opportunities for the local development of comprehensive solutions, through technologies aimed at energy efficiency, which include all sectors of the city and its building diversity.
\end{abstract}

Keywords: building diversity; energy efficiency; building regulations; envelope technologies 


\section{Introducción}

El presente trabajo se encuentra inscrito dentro del proyecto de investigación Eficiencia energética y producción de energía solar en edificios con Fachadas Ventiladas en climas templados, otorgado por el Consejo Nacional de Investigaciones Científicas y Técnicas (CONICET), de Argentina ${ }^{1}$, y desarrollado en el Instituto de Ambiente, Hábitat y Energía, de Mendoza, Argentina.

La fachada ventilada es una opción de envolvente cuya industrialización y comercialización han crecido significativamente en los países desarrollados, dado que sus características en un contexto tecnológico de alto nivel, en general, han mejorado sustantivamente la eficiencia energética de los edificios implantados en climas de alta radiación solar. Se trata de un sistema de cerramiento opaco compuesto por dos capas: una interior (de albañilería tradicional o liviana) con aislación térmica por el exterior; una exterior (placas de juntas abiertas o cerradas), y entre ambas, una cámara ventilada. Su desempeño comprende una mejora frente a una fachada convencional en cuanto al comportamiento térmico de los espacios interiores - tanto en verano como en invierno-, mediante la ventilación natural de la cámara, en la que el aire exterior accede libremente. Al respecto, el trabajo de Patania et al. (2010) indica en verano una reducción del flujo de calor que ingresa al edificio superior al $40 \%$, en comparación con la misma fachada sin ventilar. En el caso del invierno, Peci López y Ruiz de Adana Santiago (2015) demuestran que la combinación de la incidencia de radiación solar en la fachada, sumada a temperaturas elevadas y velocidades del aire bajas en el interior de la cámara, puede alcanzar importantes ahorros energéticos para calefacción, del orden del $43 \%$. Tales resultados demuestran la eficiencia del sistema de cerramiento propuesto para zonas de climas templados con alta radiación solar y amplias diferencias estacionarias.

Por otra parte, además de las propiedades físicas y constructivas de los elementos del sistema, el movimiento de aire en la cámara es un factor importante en su desempeño eficiente. La cámara entre ambas caras crea un "efecto chimenea" provocado por el calentamiento de la capa exterior. El aumento de la temperatura produce una variación en la densidad del aire, en el interior de esta con respecto al aire del exterior, con el consiguiente movimiento ascendente por convección natural. Asimismo, el revestimiento exterior provee protección frente a la radiación solar directa; para

1 El proyecto otorgado (Resolución N. 1880/2018) se encuentra en el marco de la presentación a la Carrera de Investigador Científico y Tecnológico $(\mathrm{CIC})$ de CONICET, de la Dra. Arq. Julieta Balter su mayor efectividad, se debe garantizar la ventilación de la cámara evitando su sobrecalentamiento. En este sentido, los antecedentes muestran que, si bien las principales variables que inciden en dicho movimiento son la radiación solar y la velocidad del aire exterior, el ancho de la cámara también es influyente (Balocco, 2002). De todos modos, muchos trabajos coinciden en que es importante realizar un análisis detallado del contexto previo antes de encarar un proyecto nuevo o de rehabilitación (Aparicio et al., 2014; Elarga et al., 2015; Ibáñez-Puy et al., 2017; Peci López et al., 2012; Sanjuán et al., 2011). Para esto, deben tenerse en cuenta el clima local, el diseño específico del cerramiento, las diferencias físicas y operativas de la construcción —ubicaciones de entrada y salida de aire, espesor de la cámara, propiedades físicas de los materiales-, el uso y el confort deseado del edificio, así como el costo de la energía primaria y las emisiones de $\mathrm{CO}_{2}$. Por otra parte, para que el sistema de cerramiento tenga un mejor desempeño, se debe disponer de un material con aislación térmica continua en la totalidad de la cara exterior del cerramiento interior.

El éxito del sistema en países de Europa nos permite plantear una serie de preguntas respecto a qué posibilidades de inserción tiene la fachada ventilada en el contexto latinoamericano, en el cual, si bien hay grandes urbanizaciones, los principales ejes que movilizan el desarrollo urbano son la rentabilidad económica y la elección de tecnologías constructivas asociadas a esta lógica, más que el uso de tecnologías de mayor eficiencia. Esto promueve que muchas veces se implementen tecnologías no adecuadas a los contextos. Al respecto, Pradilla Cobos (citado en Delgadillo, 2013) indica algunos riesgos de la importación indiscriminada y acrítica de conceptualizaciones urbano-edilicias que se construyeron en contextos con otras realidades socioeconómicas, y que se han impuesto a la realidad latinoamericana. Uno de ellos está dado por
[...] la parcelación del conocimiento, cada vez mayor, en pequeños compartimentos que puede que expliquen hechos puntuales de la realidad, pero pierden toda referencia a la totalidad concreta, a la construcción de expli- caciones coherentes en el ámbito del conoci- miento de la complejidad de nuestras metró- polis. (Delgadillo, 2013, p. 188)

La transferencia de tecnología es criticada también como práctica y concepto, ya que, según Salas (2000), debería denominarse

[...] importación-exportación o compra-venta de tecnología". En cualquier caso, ese mercado de tecnologías nace de la desigualdad existente entre los que la poseen y los que no la tienen. A nivel macro, la transferencia surge de la desigualdad entre países desarrollados y subdesarrollados. (p. 55) 
En relación con el aumento de densidad edilicia en las ciudades, el incremento de construcciones de gran altura de perímetro libre (torres retiradas de las líneas divisorias de edificación) en ciudades de América del Sur, basado en la adopción de modelos de Norte América y Europa, ha producido varias modificaciones en los microclimas urbanos, asociadas, a su vez, al aumento de demanda de energía (De Schiller, 2000; Leveratto, 1995). Asimismo, la cuestión ambiental es fundante en el enfoque del desarrollo sustentable; principalmente, en las ciudades de nuestra región, dado que el crecimiento económico y la urbanización desarrollada hoy en día —esta última, muchas veces no planificada- se traducen en sobreconsumos de energía, agotamiento de recursos y contaminación. Como expresan Cabalé Miranda y Pérez de Agreda (2017),

\section{La ciudad no es sólo un lugar donde se vive, ella se constituye en un nuevo ecosistema que puede integrarse a los demás o puede destruir- los, no sólo por las modificaciones que tienen lugar en ella, sino, por lo que genera en con- textos aledaños o distantes, al demandar de aquellos: energías y recursos para su funciona- miento. (p.15)}

El sistema de envolvente en estudio es utilizado en Europa en distintas escalas, así como también en diferentes tipologías edilicias: la tecnología de fachada ventilada ha sido incluida tanto en edificios corporativos (escala alta) como en viviendas multifamiliares (escala mediana) y viviendas unifamiliares (escala baja); sin embargo, su incipiente uso en el país —específicamente, en la región de Cuyo, donde se encuentra el caso en estudio: la ciudad de Mendoza- está orientado a grandes edificios corporativos, que buscan una imagen específica.

Según Salas (2000), para el estudio de una tecnología aplicada en un contexto y una temporalidad determinados se debe realizar un análisis del producto (know how), el estudio del mercado (en el contexto de economías subdesarrolladas), la distribución del producto, la promoción, el precio y la negociación. A su vez, determina aspectos clave para su adecuación, como el uso de materiales locales, mano de obra local y de fácil aprendizaje, el respeto cultural y movilizar economías regionales a partir de su implementación, entre otras. En este sentido, Cabalé y Rodríguez (2017) determinan que la compatibilidad de la actividad constructiva con el desarrollo sostenible se cumple cuando: las tecnologías se adaptan a cada sitio en particular; los materiales que se emplean son de procedencia (local/nacional), semielaborados o procesados industrialmente; la fuerza laboral es diversa, y el producto final integra actividad especializada con saberes menos calificados de los obreros locales y reduce el consumo de agua, áridos y energía.
A partir de lo expresado, consideramos necesario plantearse algunos interrogantes en relación con la importancia de ampliar el debate sobre las condicionantes sociotecnológicas y normativas de los procesos de urbanización en ciudades de América Latina, y para ello tomamos como disparador sobre el caso de estudio las preguntas: ¿Qué disponibilidades tecnológicas —materiales y sistemas constructivos- existen en Mendoza para poder incorporar apropiadamente los sistemas de fachadas ventiladas en sus diferentes escalas urbanas y sociales? ¿Cuáles serían sus posibles expresiones?, y ¿Qué instrumentos normativos se podrían tener en cuenta para que se posibilite la implementación de dichas tecnologías adaptadas a nuestra realidad? Es importante destacar que el estudio normativo y de disponibilidad tecnológica corresponde a una primera aproximación de análisis, a fin de derivar en elementos contundentes para identificar las formas de introducir o adecuar apropiadamente la tecnología de fachadas ventiladas en el contexto urbano-edilicio de la ciudad de Mendoza, Argentina.

\section{Materialidad edilicia en Mendoza: posibilidades de inserción de nuevas tecnologías de construcción tendientes a la eficiencia energética}

La fachada de un edificio es entendida desde su concepción más amplia como la cara visible de este, como la interface o el límite entre el espacio exterior y el interior, entre lo público y lo privado. En el plano internacional, con las tendencias arquitectónicas del Movimiento Moderno dadas a mediados del siglo XX, el nuevo lenguaje arquitectónico plantea una separación entre la estructura y la piel, o envolvente, lo cual modifica y amplía las posibilidades de resoluciones de fachadas, lo que llega a constituirse en la actualidad en un problema técnico, ambiental, estético, económico y social para resolverse en un proyecto edilicio.

En los inicios de la construcción de viviendas en Mendoza, las tecnologías de cerramiento utilizadas con preferencia han sido las constituidas con tierra cruda — tales como el adobe y la tapia-, debido a su raigambre cultural, su abundancia y su disponibilidad, así como a las facilidades de fabricación in situ y su forma de manufactura económica y práctica. Además, estos materiales han respondido a las condiciones climáticas de la región (frío continental con grandes amplitudes térmicas estacionales y diarias), donde las características de inercia térmica de las materialidades pesadas y macizas conforman una barrera que atenúa las condiciones extremas exteriores; sin embargo, a partir de un terremoto que destruyó la ciudad en 1861, la condición sísmica de la zona hizo que las bondades de estos materiales fueran desestimadas en las 




Adobe. Viviendas tradicionales



Ladrillo cocido. Primeras viviendas colectivas en Mendoza

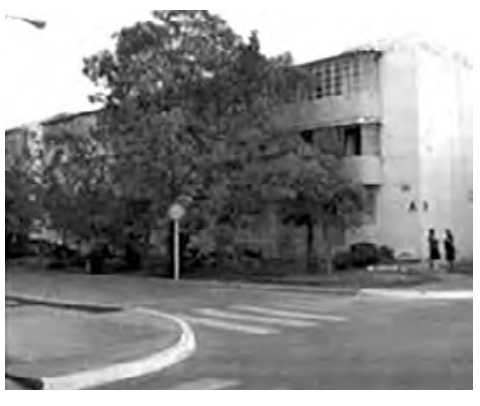

hi

\section{(1)} sin Obra Derivada. CC BY-

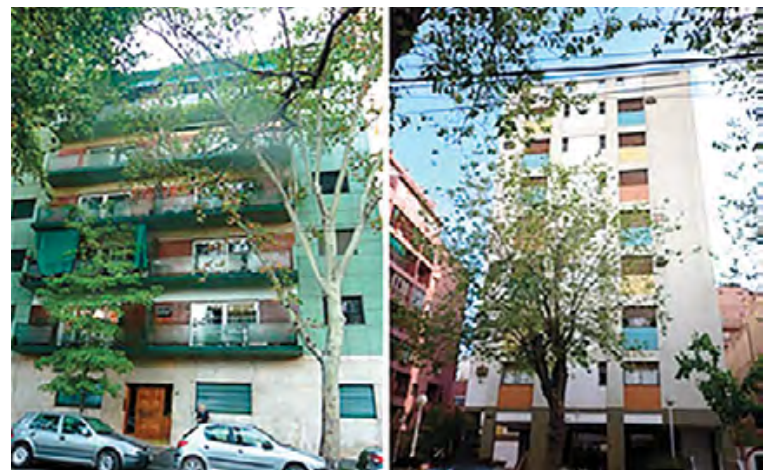

Ladrillo cerámico hueco. Primeros edificios en altura residenciales

reglamentaciones edilicias. El primer reglamento para la construcción, dictado en 1902, establecía que los muros de fachada debían ser de ladrillo o piedra asentada a la cal; además, los muros de adobe no podían superar la altura de la primera planta, y debían tener espesores de 0,40 m, 0,45 m o 0,60 m. En 1927 se amplió este reglamento, al prohibirse definitivamente los muros de adobe y determinarse espesores reglamentarios mínimos para muros de ladrillo cocido en horno: de 0,45 m para muros sin armadura; 0,30 m, para muros armados, y 0,24 m, para muros de hormigón armado (Ponte, 2008). Así fue como desde mediados del siglo XX comenzó a erradicarse la existencia de edificios construidos con adobe, y a este se lo empezó a concebir como un material despreciable y riesgoso. La fecha coincide con el terremoto de San Juan (vecina provincia ubicada al norte), en 1944, y que provocó una alerta en la provincia de Mendoza, y en particular, en el departamento de Ciudad, con la consiguiente regularización de la situación a través de normas que promovieron la erradicación del adobe en futuras construcciones, como la 3824-1944 (Miranda Gassull, 2015).

De esta manera, el riesgo sísmico de la región fue el argumento visible que llevó a instalar las soluciones constructivas del ladrillo cocido y del hormigón armado en la ciudad, como se ilustra en la figura 1.

A mediados del siglo $X X$, el crecimiento de densidad urbano en Mendoza, se vio acompañado por la construcción de edificios de altura media (entre 5 y 8 niveles), realizados en su mayoría con estructura de hormigón y envolvente de ladrillo cerámico hueco. Llegado el
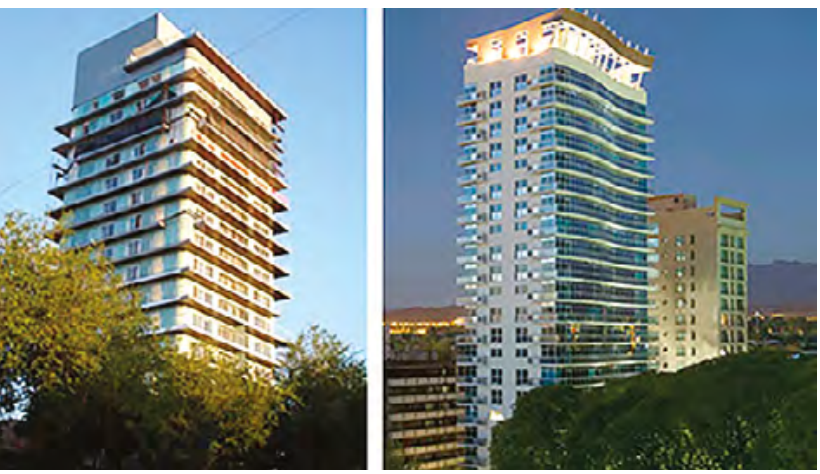

Estructura de $\mathrm{H}^{\mathrm{O}} \mathrm{A}^{\mathrm{O}}$ y envolvente liviana y transparente en edificios del siglo XXI

siglo XXI, el auge de la construcción dado en el periodo entre 2003 y 2005 (DEIE, 2007), junto a la especulación inmobiliaria, dio lugar a la construcción de edificios en torre en la zona central de la ciudad, que superan ampliamente las alturas mínimas reguladas por el código de edificación. Estos cuentan con una estructura de hormigón, y en la gran mayoría de los casos resuelven su envolvente con materiales transparentes, como se muestra en la figura 2.

En cuanto a la aceptación social de nuevas materialidades de envolvente, el sistema húmedo sigue constituyéndose en el sistema tradicional de la construcción, y a pesar de los beneficios atribuidos a la construcción en seco en cuanto a los tiempos de ejecución de proyecto - lo cual permite disminuir costos-, la resistencia hacia la incorporación de nuevas tecnologías continúa siendo fuerte. Sin ir más lejos, desde el directorio de la Cámara de Empresas Constructoras Independientes de Mendoza (CECIM) se argumentó que la problemática se debe a motivos más culturales que técnicos, debido a la dificultad de apertura de la sociedad hacia nuevas modalidades de construcción, por sentimientos de inseguridad en relación con los riesgos sísmicos (Unidiversidad, 2019).

Respecto a la implementación del sistema de fachada ventilada en Mendoza, dicha tecnología ha sido utilizada en edificios corporativos de alto costo, accesibles para un porcentaje menor de la población; por esto, es necesario pensar en maneras de adaptar la tecnología disponible en la región, en función de aprovechar los beneficios termoenergéticos que estos nuevos sistemas pueden brindar, y que, al mismo tiempo, sea una NC-ND

\& Figura 1. Tipos de construcciones formales en Mendoza a finales del siglo XIX y principios del XX.

Fuente: Ponte (2008). Licencia Reconocimiento no Comercial

Figura 2. Edificios residenciales en altura en la ciudad de Mendoza. Fuente: elaboración propia (2015). Licencia Reconocimiento no Comercial. CC BY-NC 




Ubicación de los casos de análisis en la ciudad de Mendoza


A Figura 3. Casos de estudio simulados con EnergyPlus, ubicados en Mendoza. Fuente: elaboración propia (2021). Licencia Reconocimiento no Comercial. CC BY-NC tecnología accesible para la sociedad. Dado el contexto actual podemos preguntarnos qué posibilidades tenemos de insertar nuevas tecnologías, como la de fachadas ventiladas, en el medio local. Para responder a esta pregunta se desarrollan dos dimensiones de análisis que buscan responder, por un lado, si existe promoción para la implementación de nuevas tecnologías desde los dispositivos jurídicos y normativos existentes en la ciudad de Mendoza. Y, por otro, cuáles son las reducciones en los consumos energéticos para climatización que el sistema implica (en comparación con la construcción tradicionalmente empleada), y cuáles son los costos económicos de los materiales necesarios para adaptar la tecnología local disponible al sistema de fachada ventilada (en comparación con el sistema importado por empresas extranjeras).

\section{Metodología}

El estudio abarca dos contextos de análisis: en una primera instancia, se describen los orígenes y las funciones de la fachada ventilada en Europa. Con base en el relevamiento y el monitoreo de edificios construidos en la ciudad de Barcelona, España (Balter et al., 2019), se analizan los resultados en contraste con lo regulado por el Código Técnico de Edificación de España (CTE).

En una segunda instancia, en el contexto de la región de Cuyo, Argentina, se analiza el caso local de la ciudad de Mendoza, en función de abarcar dos ejes: el análisis normativo provincial y el estudio económico-energético de la tecnología existente en el mercado.

El aspecto normativo analiza en qué medida los dispositivos jurídicos locales promueven, adaptan o adoptan (o no) el acceso masivo a las nuevas tecnologías. Se toma como referencia el Código de Edificación de la ciudad de Mendoza. Este contiene un conjunto de ordenanzas y directrices que integran y orientan la estructura discursiva que organiza la construcción urbana en el departamento de capital. También se debe tener en cuenta que dicho código es la base de referencia para la reglamentación de la edificación en otros municipios.
Para abordar la problemática planteada e incorporarla en el marco normativo, consideramos necesario establecer dispositivos jurídicos que reglamenten su complejidad. Según Foucault (2005), la noción de dispositivo (dispositif) se aborda como una herramienta teórica de análisis que reconstruye la relación entre saber y poder, lo dicho y lo no dicho, y la heterogeneidad de componentes. En este sentido, Vega (2017) define que "Estas cualidades permiten al dispositivo tener un emplazamiento histórico y responder a urgencias específicas de un tiempo y espacio singulares" (p. 140). En el presente caso de estudio se aborda el dispositivo como un procedimiento y una serie de normativas que rigen el uso y el acceso de la heterogeneidad de propuestas tecnológicas en la construcción. Se conocen como dispositivos jurídicos aquellos elementos normativos (como leyes, ordenanzas, normas) y procedimentales (códigos de edificación, Etiquetado de Eficiencia Energética Edilicia) que permiten identificar las relaciones poder-hacer entre las tecnologías que se encuentran en el marco legal-formal y aquellas que también abundan en nuestra realidad, donde quedan en la práctica no discursiva y se traducen en lo que denominamos informalidad constructiva.

Por otro lado, se realiza una primera aproximación de análisis de disponibilidad tecnológica existente en Mendoza, para poder incorporar apropiadamente los sistemas de fachadas ventiladas en sus diferentes escalas urbanas y sociales. En este eje se analizan el aspecto económico y el energético: el primero se estudia en función de comparar los costos (por $\mathrm{m}^{2}$ ) de tecnologías accesibles en el ámbito local y posibles de adaptar al sistema de fachada ventilada, en contraposición al sistema disponible en Mendoza, pero importado y comercializado por empresas extranjeras. Para tal fin, se relevan y se consideran presupuestos técnico-económicos solicitados a empresas locales y distribuidoras de los sistemas importados.

El aspecto energético se analiza de acuerdo con estudios previos de rehabilitación edilicia en alta densidad en Mendoza (Balter et al., 2020), en complemento con el análisis de un caso de 
estudio en baja densidad, ubicado en un barrio popular localizado en el área pedemontana de la ciudad de Mendoza, según se muestra en la figura 3. Se trata de una vivienda autoconstruida por una familia tipo, de $35 \mathrm{~m}^{2}$ cubiertos, con cocina-comedor, una habitación, un baño y el espacio disponible para futuras construcciones, destinadas a una segunda habitación $\left(16 \mathrm{~m}^{2}\right)$ y un patio interno $\left(14 \mathrm{~m}^{2}\right)$. La construcción fue realizada con muros de ladrillo a la vista en el interior y pintura (sin revoque) por el exterior, y cubierta a dos aguas de rollizos, machimbre y membrana.

En ambos casos (alta y baja densidad) se trabajó con modelos de simulación dinámica, mediante el programa EnergyPlus, versión 8.7². Las simulaciones se realizaron en verano tomando periodos de 20 días e ingresando el archivo climático (extensión EPW) con los datos requeridos. Se evaluaron los consumos energéticos $(\mathrm{kWh})$ necesarios para estar en confort (en verano, $24{ }^{\circ} \mathrm{C}$ ) del caso existente, en comparación a los resultados de incorporar el sistema de fachada ventilada en la envolvente. Para esto, se utilizó, dentro del módulo de construcciones avanzadas, el objeto de "Cavidad exterior naturalmente ventilada" (Exterior Natural Vented Cavity), utilizado para trazar una capa separada de la capa interior, lo que permite definir las características de la cavidad y las aberturas para superficies exteriores con ventilación natural, como se detalla en la tabla 1. Este objeto se utiliza junto con el modelo de "Otras condiciones laterales" (Other Side Conditions Model), donde se configuró la opción de "Espacio de convección por radiación" (Gap Convection Radiation), la cual proporciona las condiciones de contorno para la convección y la radiación térmica de la cámara de ventilación modelada por separado a la superficie de la envolvente. En el caso de la vivienda unifamiliar, la fachada ventilada se incorporó en todos los muros exteriores de la vivienda; es decir, hacia todas las orientaciones.

\section{Resultados}

\section{Orígenes y funciones de la fachada ventilada en Europa: estudio de casos en Barcelona}

Los orígenes de la doble piel en la envolvente de los edificios se remontan a fines del siglo XIX, en el Reino Unido, con el Cavity Wall. Este sistema nace en medio de la corriente higienista de la época y en un contexto climático de precipitaciones persistentes, y donde las fachadas tra-

2 Programa gratuito desarrollado por el Laboratorio Nacional Lawrence Berkeley (LBNL); es actualmente el software oficial para la simulación del Departamento de Energía de Estados Unidos.

dicionalmente eran construidas con materiales porosos. Se trata de una fachada de doble hoja limitando una cámara drenante — Rain Screen-, con el objetivo de garantizar la estanqueidad al agua. Dicho sistema se adoptó en España en la década del 90 del siglo XX, renombrado como fachada ventilada. Con este cambio se pone de manifiesto el poco interés en los problemas de estanqueidad, para, en cambio, dar relevancia al comportamiento térmico de la fachada (Paricio \& Pardal, 2014).

En la actualidad, la industria de revestimientos que conforman la cara exterior de la fachada ventilada en Europa ofrece una amplia variedad de materiales, colores y formas: materiales como cerámicos, gres porcelánico, roca basalto, hormigón reforzado, hormigón polímero, placas cementicias y fenólicos reforzados. Por su parte, la hoja interior puede ser liviana o de construcción tradicional de albañilería. Al respecto, Cristina Pardal (2009) plantea una contradicción que puede encontrarse en la convivencia entre dos sistemas constructivos muy distintos: la hoja exterior - ligera, de montaje en seco y con un alto nivel de tecnificación-confía su estabilidad mediante sistemas de fijación a la hoja interior, la cual sigue resuelta con la albañilería tradicional. Esta convivencia no supondría problemas si permitiera concebir la fachada como un conjunto, pero las pocas garantías de estabilidad y planeidad de la hoja interior no permiten confiar en ella como soporte. La autora plantea el desarrollo de paneles que resuelvan la hoja interior de manera prefabricada, como un soporte de la estructura principal del edificio. El planteo de esta solución de fachada liviana conlleva satisfacer tanto los requerimientos tecnológicos como la envolvente en su conjunto, incluyendo como aportes añadidos los valores de la industrializa-

(V) Tabla 1. Elementos de entrada para la definición de la fachada ventilada en EnergyPlus.

Fuente: elaboración propia (2021). Licencia Reconocimiento no Comercial. CC BY-NC

\begin{tabular}{|c|c|c|}
\hline $\begin{array}{c}\text { Propiedades de la cavidad } \\
\text { ventilada exterior }\end{array}$ & $\begin{array}{l}\text { Unidad de } \\
\text { medida }\end{array}$ & Valor \\
\hline Fracción de área de aberturas & adimensional & 1 \\
\hline $\begin{array}{l}\text { Emisividad térmica de la placa exterior } \\
\text { (tipo placa cementicia) }\end{array}$ & adimensional & 0,9 \\
\hline $\begin{array}{l}\text { Absortividad solar de la placa exterior } \\
\text { (tipo placa cementicia) }\end{array}$ & adimensional & 0,1 \\
\hline $\begin{array}{l}\text { Aislación térmica (poliestireno } \\
\text { expandido) }\end{array}$ & $\mathrm{m}$ & 0,05 \\
\hline Altura de la cámara ventilada & $\mathrm{m}$ & 2,7 \\
\hline Espesor de la cámara ventilada & $\mathrm{m}$ & 0,1 \\
\hline $\begin{array}{l}\text { Relación entre la superficie real y la } \\
\text { superficie proyectada }\end{array}$ & adimensional & 1 \\
\hline Rugosidad de la superficie exterior & adimensional & suave \\
\hline Perforaciones respecto al viento & adimensional & 0,25 \\
\hline $\begin{array}{l}\text { Coeficiente de descarga para aberturas } \\
\text { respecto al flujo por flotabilidad }\end{array}$ & adimensional & 0,65 \\
\hline
\end{tabular}




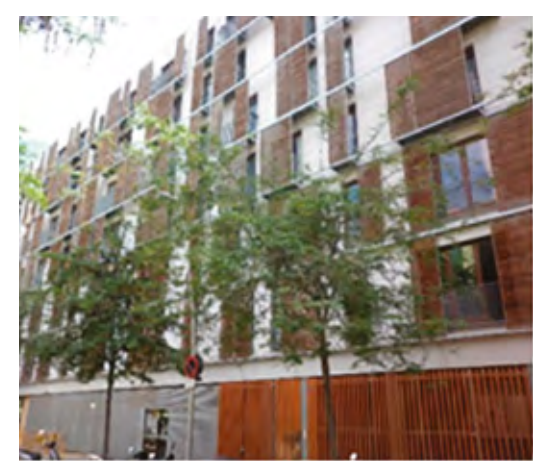

Cámara cerrada. Ventilación por juntas abiertas


Cámara cerrada. Ventilación por juntas abiertas
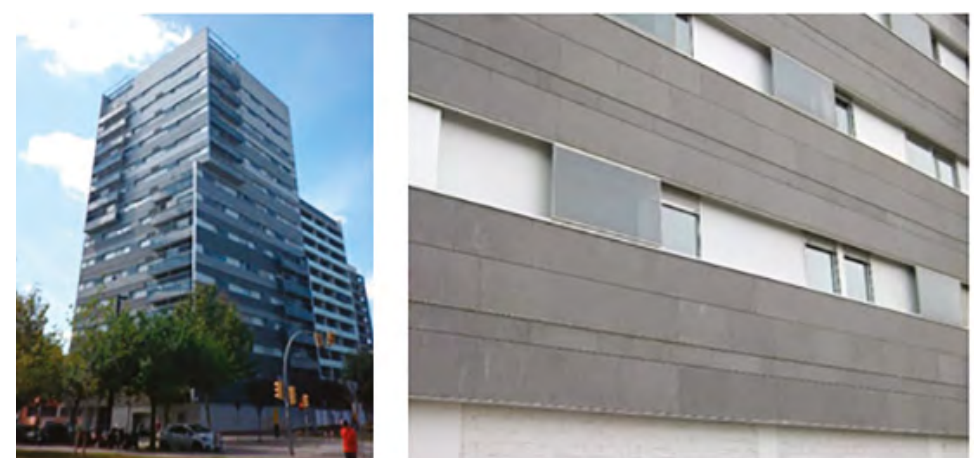

Cámara cerrada. Ventilación por juntas abiertas
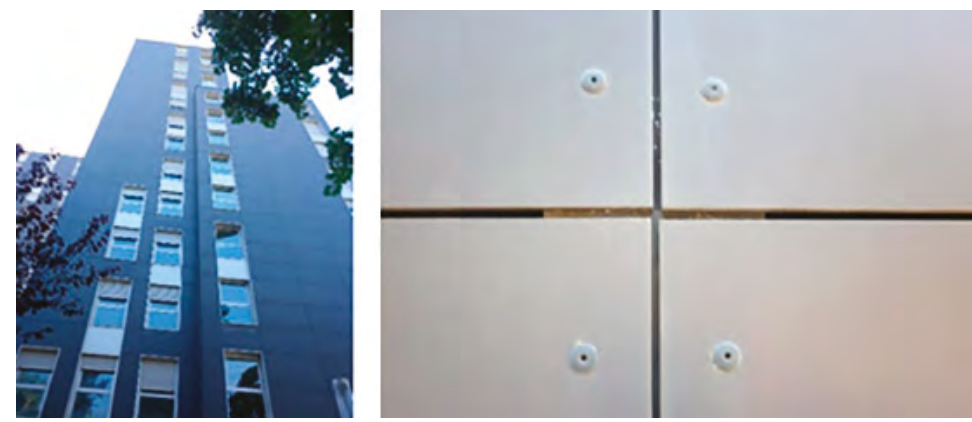

Cámara cerrada. Ventilación por juntas abiertas
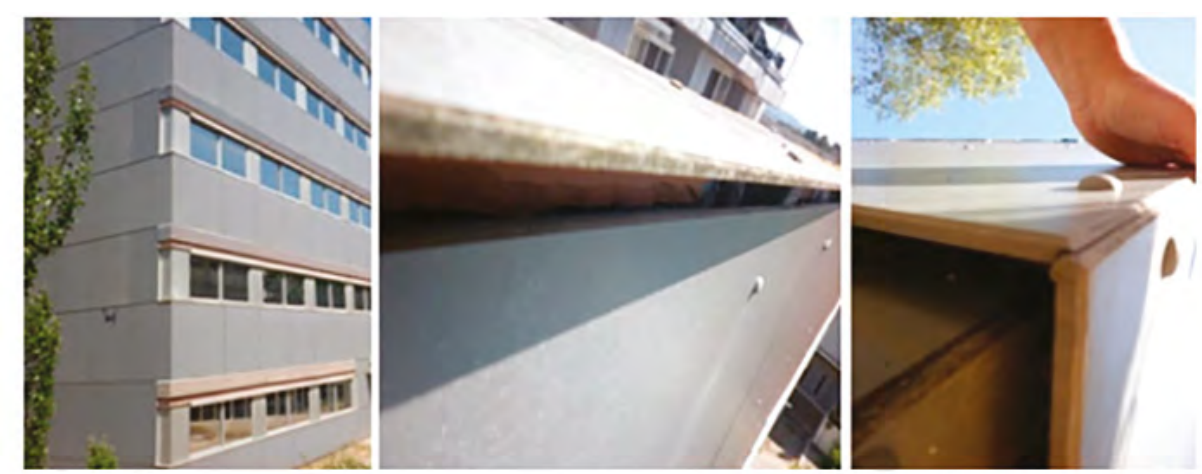

Cámara abierta. Ventilación por apertura inferior y superior
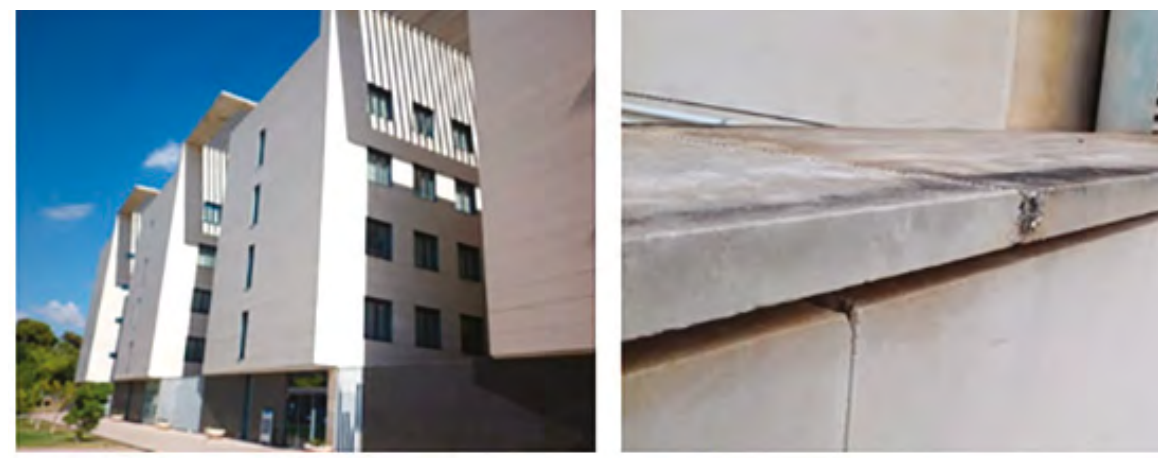

Cámara semi abierta - Ventilación por apertura superior

(A) Figura 4. Ejemplos de edificios con distintos tipos de cámara de fachada ventilada: cámara cerrada, abierta y semiabierta.

Fuente: elaboración propia (2019). Licencia Reconocimiento no Comercial. CC BY-NC ción, de la libertad compositiva y de la ganancia del espacio útil, al reducir el grueso del cerramiento (Pardal, 2009).

En cuanto a las políticas de gobierno dadas en España en relación con esta tecnología, el CTE incluye la fachada ventilada en las condiciones exigidas a cada solución constructiva de muros y fachadas; sin embargo, también exige que para cualquier solución alternativa, incluidas en el propio documento (ladrillo, hormigón o piedra natural), exista una aprobación a través de un documento reconocido que garantice el cumpli- miento de las prestaciones indicadas. El Documento de Idoneidad Técnica Europeo (DITE) es la evaluación técnica favorable de un producto para el uso asignado, concedido por organismos pertenecientes a la Organización para la Idoneidad Técnica Europea (en inglés, EOTA, por las iniciales de European Organisation for Technical Approvals). El Instituto de Ciencias de la Construcción Eduardo Torroja (IETcC) está autorizado para la concesión del DITE, y es, además, el portavoz español en la EOTA. De esta manera, cualquier propuesta innovadora que no vaya ligada a una empresa capaz de darle soporte resulta inviable económicamente.

En la ciudad de Barcelona, España, para el estudio de dichas tecnologías, se llevó a cabo un estudio de casos in situ, enfocado en el funcionamiento de la ventilación de la cámara de aire de la fachada ventilada, en relación con lo pautado por las normativas de edificación. El estudio partió de considerar que la situación real de los edificios construidos suele ser muy diferente de la de los estudios teóricos (académicos) realizados por simulación computacional o numérica (Balocco, 2002; 2004; Domínguez Delgado et al., 2013; Patania et al., 2010; Suárez \& Molina, 2015), en los cuales no se consideran los sistemas de fijación de la hoja exterior, elementos que se encuentran regularmente dispuestos dentro de la cámara y pueden interceder en el movimiento del aire en su interior.

En función de entender y validar las predicciones del comportamiento del sistema, se realizaron mediciones de velocidad y temperatura del aire en el interior de la cámara de diez casos de estudio, con cámaras que ventilan por las juntas 


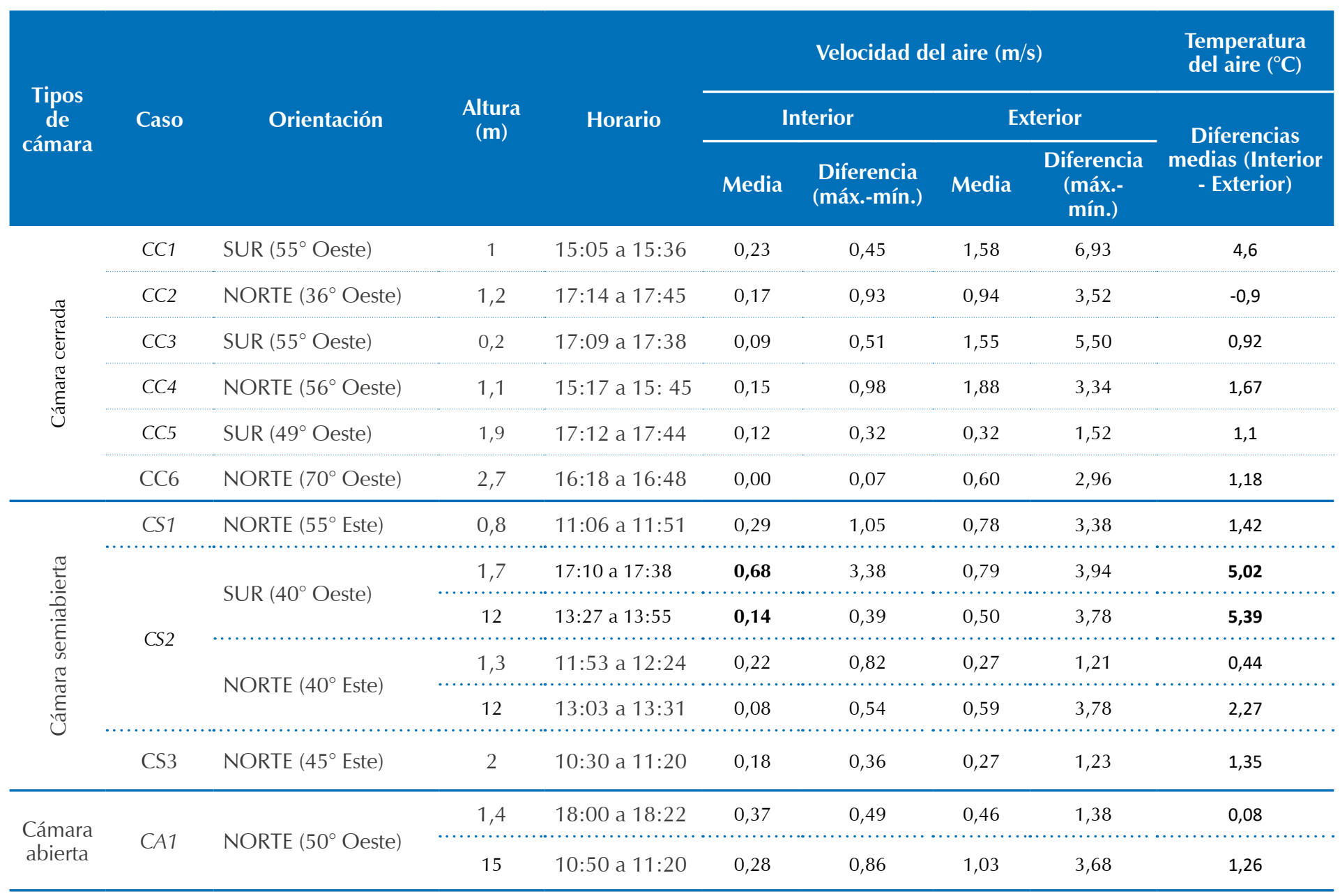

abiertas y casos que ventilan por las aperturas superiores o las inferiores, tal cual se muestra en la figura 4.

La información obtenida, según se ve en la tabla 2, se contrastó con los aspectos regulados por el CTE en cuanto a la clasificación de las cámaras de aire y su grado de ventilación. Al respecto, el CTE regula la ventilación de la cámara considerando un área mínima de ventilación por cada metro cuadrado de fachada, con lo cual avala su ventilación a través de las juntas abiertas de las hojas exteriores, y no considera las aperturas inferiores y superiores de la cámara. Los resultados de las mediciones realizadas indicaron un mejor desempeño del sistema en función del aumento de las aperturas inferiores y superiores de aire en la cámara, variable que no se regula en el código. Por ejemplo, la velocidad de aire registrada en los casos con apertura en los extremos inferiores y superiores de la cámara (CS y CA en tabla 2), resultó significativamente mayor (en el orden del $80 \%$ ) a la velocidad del aire en los casos que solo ventilan por las juntas abiertas. Un ejemplo clarificador del funcionamiento de la cámara ventilada es el caso CS2, que cuenta con la apertura inferior de la cámara abierta, y con la apertura superior cerrada. Se puede observar que la medición realizada en la cara Sur-Oeste del edificio (hacia el ecuador terrestre en el Hemisferio Norte) muestra los mayores aumentos de temperatura en la cáma- ra - del orden de los $5{ }^{\circ} \mathrm{C}$-, lo cual hace que el aire se mueva con mayor velocidad $-0,68$ $\mathrm{m} / \mathrm{s}$ - en la sección de la cámara más cercana a la apertura (medición a 1,7 m de altura); sin embargo, en la parte superior los resultados indican un calentamiento del aire de $5,4{ }^{\circ} \mathrm{C}$, pero al estar cerrada la cámara en su extremo superior (medición a $12 \mathrm{~m}$ de altura) el aire se mueve a menor velocidad: en el orden de los 0,14 m/s.

Además de lo anterior, en todos los casos monitoreados la superficie de juntas abiertas de la hoja exterior excede considerablemente el valor mínimo requerido por la normativa para que la cámara sea ventilada. Se observan, entonces, significativas variaciones existentes entre lo regulado por el código y lo efectivamente construido, tanto en la ventilación de la cámara como en las características físicas y geométricas de sus elementos.

\section{Análisis normativo y de disponibilidad tecnológica en la ciudad de Mendoza, Argentina}

Los dispositivos jurídicos como instrumentos para guiar condiciones constructivas y sociales

El conjunto de normas en Mendoza que regulan las formas, los métodos, los procedimientos y las costumbres del quehacer constructivo son entendidas como dispositivos jurídicos, y constituyen el instrumento para guiar las condiciones
Tabla 2. Resultados del monitoreo in situ en casos de estudio en Barcelona. Fuente: elaboración propia (2021). Licencia Reconocimiento no Comercial. CC BY-NC 
específicas de los nuevos sistemas tecnológicos/ constructivos en función de considerar los contextos sociourbanos.

Se toma como referencia de dispositivo jurídico el Código Urbano y de Edificación de la Ciudad de Mendoza (2019). Este cuenta con el capítulo E.II. Normas Constructivas Generales Propias de los Edificios, que establece, en el apartado E.II.6. De los Materiales y Sistemas Constructivos, las posibilidades de inserción de nuevas tecnologías y sistemas en el medio local, y en el cual se desarrollan los siguientes cinco puntos:

1. Materiales y sistemas permitidos: Se establece que solo pueden utilizarse los aprobados por el Departamento Ejecutivo, bajo asesoramiento de la Comisión de Estudio de Nuevos Materiales y la conformidad del Ministerio de Obras y Servicios Públicos de la Provincia.

2. Calidad de los materiales de la construcción e instalaciones: Se establecen las condiciones de calidad de los materiales, donde la Dirección de Obras Privadas podrá impedir el empleo de productos que juzgue impropios, mediante resoluciones internas o normas especiales aprobadas por el Departamento Ejecutivo.

3. Comisión de asesoramiento para la aprobación de nuevos materiales y sistemas constructivos: Se establecen los miembros representantes de distintas entidades (Ministerio de Obras Privadas y Servicios Públicos; Consejo Profesional de Ingenieros, Arquitectos, Agrimensores y Geólogos de Mendoza; Dirección de Obras Privadas, y Dirección de Obras Municipales de Capital) designados por los respectivos organismos.

4. Obligación de cumplir las normas sobre materiales y sistemas: Se establece que quien solicite la aprobación de un material o un sistema contrae el compromiso de actuar de conformidad a los términos concedidos, y que el Departamento Ejecutivo, al aprobar un sistema, "no contrae obligación de mantener el empleo de los mismos, pudiendo disponer modificaciones o supresiones de un sistema, material, producto de la industria y cualquiera de sus partes, anulando parcial o totalmente la aprobación acordada si lo juzga necesario" (Código Urbano y de Edificación de la ciudad de Mendoza, 2019).

5. Tramitación para aprobación de sistemas constructivos no tradicionales: Se establece que solo pueden solicitar la aprobación de un sistema las empresas o las sociedades civiles, comerciales o industriales, representadas por un profesional habilitado. En este punto se detalla la documentación para presentar (memoria descriptiva, certificados de ensayos y aprobatorios por entes oficiales, documentación gráfica con especificaciones, detalles de la ejecución de un prototipo, descripción del proceso de fabricación o ejecución, etc.). Asimismo, en este apartado el código especifica que toda aprobación provincial de sistemas constructivos no tradicionales se hará por ordenanza general, y autorizando solamente la construcción de hasta 20 unidades de vivienda, con inspecciones periódicas, hasta los dos años contados desde la certificación final de la primera de las viviendas. Luego de esto, el fabricante y su representante legal podrán solicitar su aprobación definitiva.

A partir de lo expuesto, se observa en la lectura del código de edificación un grado de complejidad significativo en relación con la inclusión de nuevos sistemas tecnológicos, tanto en los procedimientos como en los aspectos técnicos. Esto dificulta la incorporación de tecnologías intermedias que adhieran a la eficiencia energética en la baja y mediana escala edilicia y sean accesibles en el contexto socioeconómico actual.

Cabe destacar que la Municipalidad de Capital de Mendoza incorporó en la Ordenanza 3978/19 —en el capítulo U.II.4.1.4. Construcciones Sustentables - mínimos obligatorios de sustentabilidad en relación con tres aspectos: 1) la incorporación de sistemas de control solar exterior en el $100 \%$ de los aventanamientos; 2) materiales en superficies exteriores horizontales con valores de albedo en el rango de 0,8-1 (es decir, colores claros), con el fin de mitigar la alta absorción de temperatura, y 3) la creación de áreas verdes para favorecer el enfriamiento y la ventilación. La ordenanza establece seis categorías ambientales de sustentabilidad, donde los proyectos que alcancen el mayor puntaje podrán tener el beneficio de aumentar el Factor de Ocupación Total (FOT), entendido como la superficie total edificable en el terreno, hasta el $25 \%$; o bien, se puede optar por una retribución económica para ser utilizado en obras de mejoramiento urbano del entorno de la obra que se va a construir.

Con esta ordenanza se observa la intención de promover la aplicación de estrategias sustentables; sin embargo, si bien se precisa un rango de albedo para las superficies exteriores, no existe una regulación respecto a los porcentajes de envolvente opaca y transparente, aspecto que resulta determinante para el desempeño termoenergético de los edificios expuestos a la alta radiación solar de Mendoza (Balter et al., 2016). Asimismo, con el incentivo del aumento de la superficie total por construir se corre el riesgo de que este sea un fomento para los intereses inmobiliarios, más que para prácticas arquitectónicas responsables con el medio ambiente. El aumento del FOT en un proyecto de grandes dimensiones y gran altura puede no solo perjudicar la habitabilidad de las construcciones vecinas —en relación con la pérdida de acceso al sol, a la luz natural y a la privacidad-, sino que también 




puede disminuir las posibilidades de enfriamiento convectivo y radiativo en el centro de la ciudad, y aumentar así el fenómeno de isla de calor en la ciudad (Correa, 2006).

A partir de lo expuesto, se observa que las dificultades mencionadas pueden atentar contra aquellas ideas que pueden dar respuestas concretas en cuanto a la mejora en la habitabilidad, entendiendo que, por ejemplo, la tecnología objeto de este trabajo puede comenzar a adaptarse y formar parte también de las estrategias para implementar en la mediana y la baja escala edilicia. En consecuencia, se considera necesario generar estructuras dentro de estos marcos jurídicos que permitan facilitar y agilizar dichos procedimientos de validación, a efectos de poder implementar en lo mediato tecnologías apropiadas, orientadas a minimizar el consumo energético y mejorar las condiciones de habitabilidad en un contexto socio-edilicio demandante de mejoras estructurales.

Aspectos económicos y energéticos de sistemas de fachada ventilada disponibles en Mendoza

Se presenta un análisis de los materiales disponibles en Mendoza factibles de ser adaptados como fachada ventilada, en contraste con los sistemas importados que pueden adquirirse. Un material posible de adaptar a las fachadas ventiladas, y que es, además, de fabricación nacional, son las placas cementicias para exterior, de 8 y $10 \mathrm{~mm}$ de espesor, con distintas opciones de texturas, colores y terminaciones. En la tabla 3 se muestran los costos presupuestados (febrero-marzo de 2020) de los materiales para la hoja exterior -incluida la estructura portante de las placas - junto a diferentes opciones de materiales de aislación térmica necesarias para el correcto funcionamiento del sistema.
El costo económico de las placas cementicias en estudio -incluyendo la estructura portante completa - se encuentra en el orden de los 36,5 dólares (marzo 2020) el metro cuadrado de construcción. Las otras opciones de fachada ventilada las proveen empresas internacionales que tienen representantes en la provincia de Mendoza e importan totalmente sus sistemas, y cuentan con una amplia gama de diseños. En este caso, los valores presupuestados —en los que se incluye también la estructura portante- superan diez veces el costo de las placas cementicias nacionales.

Respecto al comportamiento energético, se presentan los resultados obtenidos en un edificio residencial de siete niveles de altura en Mendoza. El caso de estudio fue analizado mediante monitoreo de las condiciones térmicas in situ, validación de modelos dinámicos mediante EnergyPlus y su posterior rehabilitación mediante la incorporación de Fachada Ventilada (Balter et al., 2020). Los resultados indicaron una disminución de cargas energéticas para refrigeración — considerando $24{ }^{\circ} \mathrm{C}$ interiores-, del orden del $1 \%$ como promedio del total del edificio. Estos porcentajes moderados se ven afectados por el carácter compacto de las viviendas, donde solo el $12 \%$ del total de la envolvente de cada unidad se encuentra expuesta al Norte; sin embargo, al incorporar ventilación en la cubierta de la vivienda del último nivel —ubicada en el séptimo piso-, los resultados promedio del total del edificio muestran mejoras energéticas del $32 \%$.

En cuanto a la vivienda unifamiliar analizada en este trabajo, la incorporación de aislación térmica y revestimiento exterior cementicio en la envolvente implicó una reducción en la transmitancia térmica de muros del $75 \%\left(4,3 \mathrm{~W} / \mathrm{m}^{2 \circ} \mathrm{C}\right.$ caso base; $1,1 \mathrm{~W} / \mathrm{m}^{2 \circ} \mathrm{C}$ caso con fachada ventilada). Los resultados de la simulación dinámica del
A Tabla 3. Materiales y tecnologías posibles de ser utilizadas como Fachada Ventilada en Mendoza. Fuente: elaboración propia (2020). Licencia Reconocimiento no Comercial. CC BY-NC 
comportamiento interior muestran una reducción de los consumos mensuales necesarios para estar en confort en verano del 18,8\%. En comparación a la disminución del $1 \%$ de las viviendas ubicadas en un edificio en altura, se puede observar que la vivienda analizada, al contar con una envolvente totalmente expuesta al exterior, resulta más sensible a los beneficios termoenergéticos que puede aportar la fachada ventilada para el clima específico de Mendoza.

\section{Discusión}

El presente trabajo explora las posibilidades de introducir y adecuar desde la formalidad normativa, administrativa y tecnológica el sistema de cerramiento de fachada ventilada en ciudades de nuestra región, en función de disminuir los consumos energéticos edilicios, a partir de soluciones locales e integrales, tanto para la rehabilitación edilicia como para edificios nuevos.

El análisis advierte que la apertura hacia nuevas tecnologías de envolvente no es un desafío fácil en nuestro contexto regional ni, en particular, en Mendoza. Los dispositivos jurídicos expresados a través de normativas y procedimientos se presentan como elementos complejos, donde solo los sectores residenciales de alta gama y de edificios corporativos o administrativos pueden acceder a sus beneficios.

Respecto al estado edilicio y de construcción de los últimos años en Mendoza, las nuevas tecnologías de envolvente, expresadas como objetos importados de países "desarrollados", totalmente desvinculadas de lo local, pueden también representar estas manifestaciones territoriales de la desigualdad social existente en la ciudad.

En cuanto a las mediciones efectuadas en los casos de estudio de la ciudad de Barcelona, se advirtió que la orientación al ecuador terrestre tiene el mejor desempeño en cuanto a valores de velocidad y caudal de aire en la cámara ventilada, lo cual es coincidente con otras investigaciones realizadas (Stazi et al., 2011). La situación en España, si bien representa otro contexto sociotecnológico y económico, muestra un éxito en la inclusión de sistemas de fachadas ventiladas en un amplio sector edilicio: la tecnología ha sido implementada tanto en el sector residencial como en el comercial-administrativo y el hotelero, abarcando todas las escalas. Pero a pesar de ello, se observan importantes diferencias entre lo regulado jurídicamente y la efectiva implementación y el efectivo funcionamiento del sistema.

En el área local, respecto al marco jurídico de Mendoza, las nuevas tecnologías con niveles de innovación encuentran limitaciones para cumplimentar los requisitos normativos, ya que no siempre se cuenta con los respaldos de las instituciones involucradas para su aprobación definitiva. En este contexto, solo las tecnolo- gías formales - en nuestro caso, gran parte de ellas son de origen extranjero- responden a los requerimientos, pero, en general, son de implementación selectiva, dados su escala y sus altos costos. Las posibilidades de incluir otras tecnologías viables que puedan generarse o adaptarse para ser implementadas en la mediana y baja escala edilicia son limitadas. Esto se debe a la multiplicidad de barreras para su aprobación, entre las cuales se debe pasar por diversos ensayos; además, si la propuesta no está vinculada con alguna empresa que pueda darle soporte, resulta inviable económicamente.

En cuanto a los materiales posibles de ser utilizados como placas exteriores de industria nacional, las opciones son escasas; no obstante, considerando que es posible una reducción de los consumos energéticos para climatización del orden del $20 \%$, si se toman en cuenta los aspectos constructivos determinantes para el efectivo funcionamiento del sistema de cerramiento - como la aislación y las aperturas inferiores y superiores de la cámara-, la tecnología local disponible puede resultar una solución adecuada para la escala media y baja de la ciudad. Asimismo, las diferencias en cuanto a costos respecto a la tecnología importada superan el $1000 \%$, lo cual indica importantes oportunidades para el desarrollo local de estas tecnologías tendientes a la eficiencia energética.

\section{Conclusiones}

El presente trabajo muestra las potenciales oportunidades de adaptar la tecnología disponible en Mendoza para poder incorporar la fachada ventilada en sus diferentes escalas y expresiones, mediante soluciones integrales que incluyan a todos los sectores de la ciudad y su diversidad constructiva.

El sistema de envolvente en estudio resulta una opción adecuada en climas con alta radiación solar, como es el caso de la región de Cuyo: la incorporación de la fachada ventilada en unidades de vivienda ubicadas en Mendoza muestra importantes reducciones en los consumos energéticos para climatización.

En relación con el análisis económico, las alternativas de adaptación de la materialidad existente en el ámbito local disminuyen sustancialmente, en comparación al sistema importado que se encuentra disponible en Mendoza. La materialidad existente, a su vez, es conocida y manipulada regularmente por la mano de obra local, a diferencia de los sistemas importados.

En cuanto a los marcos jurídicos, se observa la potencial necesidad de flexibilizar algunas ordenanzas del Código Urbano y de Edificación de la Ciudad de Mendoza, de forma que permita habilitar una mayor diversidad de tecnologías alternativas, entre las que se considera a 
la fachada ventilada una opción válida y eficaz para el contexto urbano-edilicio de análisis. Por otra parte, las políticas de fomento de sustentabilidad dadas en dicha normativa están mayormente orientadas a mitigar los efectos de isla de calor urbana; sin embargo, no se observan requerimientos precisos orientados a mejorar las condiciones termoenergéticas interiores de las construcciones, por lo que se recomienda incorporar aspectos que se refieran a las características de opacidad y transparencia, así como a los coeficientes de transmitancia de las envolventes, de acuerdo con su exposición exterior.
Por otro lado, se considera oportuno también pensar en la aplicación de incentivos económicos a través de fondos solidarios, para que las pymes puedan acceder a los requerimientos mínimos que aprueben el uso de nuevas tecnologías. Desde el lado de la producción y la oferta tecnológica, esto podría conducir a mayores posibilidades de igualdad empresarial dando acceso y legalidad a los pequeños emprendimientos que quieran desarrollar tecnologías de envolvente con mayor eficiencia energética. Y desde el lado de la demanda, permitiría brindar alternativas tecnológicas eficientes y más accesibles a la edilicia de mediana escala.

\section{Referencias}

Aparicio-Fernández, C., Vivancos, J. L., FerrerGisbert, P., \& Royo-Pastor, R. (2014). Energy performance of a ventilated façade by simulation with experimental validation. Applied Thermal Engineering, 66(1-2), 563-570. https://doi.org/10.1016/j.applthermaleng.2014.02.041

Balocco, C. (2002). A simple model to study ventilated facades energy performance. Energy and Buildings, 34(5), 469-475. https://doi.org/10.1016/S03787788(01)00130-X

Balocco, C. (2004). A non-dimensional analysis of a ventilated double façade energy performance. Energy and Buildings, 36(1), 35-40. https://doi.org/10.1016/S03787788(03)00086-0

Balter, J., Ganem, C., \& Barea, G. (2020). Mejoras en el desempeño energético de edificios en verano mediante la integración de envolventes ventiladas en fachadas norte y cubiertas. El caso de Mendoza, Argentina. Hábitat Sustentable, 10(2), 94-105. https://doi.org/10.22320/07190700.2020.1 0.02 .07

Balter, J., Ganem, C., \& Discoli, C. (2016). On high-rise residential buildings in an oasiscity: thermal and energy assessment of different envelope materiality above and below tree canopy. Energy and Buildings, 113(1), 61-73.

https://doi.org/10.1016/j.enbuild.2015.11.011

Balter, J., Pardal, C., Paricio, I., \& Ganem, C. (2019). Air cavity performance in opaque ventilated facades in accordance with the span technical building code. ACE: Architecture, City and Environment, Arquitectura, Ciudad y Entorno, 13(39), 211-232. https://doi.org/10.5821/ace.13.39.6487

Cabalé Miranda, E., \& Rodríguez Pérez de Agreda, G. (2017). El desarrollo sostenible en la actividad constructiva. Revista de Estudios del Desarrollo Social: Cuba y América Latina, 43, 40-51. , 5(2).

Código Urbano y de Edificación de la Ciudad de Mendoza. (2019).

https://ciudaddemendoza.gob.ar/wp-content/ uploads/2020/04/Co\%CC\%81digo-Urbano-yde-Edificacio\%CC\%81n-0001-2019.pdf

Correa, E. (2006). Isla de calor urbana. El caso del área metropolitana de Mendoza [Tesis doctoral, Universidad Nacional de Salta, Salta, Argentina.]

Delgadillo, V. (2013). América Latina urbana: la construcción de un pensamiento teórico propio. Entrevista con Emilio Pradilla Cobos. Revista Andamios, 10, 185-201. http://dx.doi.org/10.29092/uacm. v10i22.272
De Schiller, S. (2000). Sustainable cities: contribution of urban morphology. Proceedings of PLEA-2000, Passive\&Low, Cambridge, United Kingdom, 353-358.

DEIE. (2007). Informe económico 2007. Mendoza. Sector construcciones. http://www. deie.mendoza.gov.ar

Domínguez Delgado, A., Durand Neyra, P., \& Domínguez Torres, C.A. (20-22 de mayo de 2013). Estudio del enfriamiento pasivo por fachadas ventiladas en el sur de España. En Actas del I Congreso Internacional de Construcción Sostenible y Soluciones Eco-eficientes. Sevilla, España, Universidad de Sevilla, 193-205.

Elarga, H., De Carli, M., \& Zarrella, A. (2015). A simplified mathematical model for transient simulation of thermal performance and energy assessment for active facades. Energy and Buildings, 104(1), 97-107. h t tp s://doi.org/10.1016/j. enbuild.2015.07.007

Foucault, M. (2005). Vigilar y castigar. Nacimiento de la prisión (Aurelio Garzón del Camino, Trad.). Siglo XXI.

Ibañez-Puy, M., Vidaurre-Arbizu, M., SacristánFernández, J., \& Martín-Gómez, C. (2017). Opaque ventilated façades: Thermal and energy performance review. Renewable and Sustainable. Energy Reviews, 79, 180-191. https://doi.org/10.1016/j.rser.2017.05.059

Leveratto, M. J. (1995). El impacto de edificios en torre de gran altura y confort en espacios urbanos. Anais III Encontro Nacional y i Encontro Latino-Americano de Conforto no Ambiente Construido, ANTAC, Porto Alegre.

Miranda Gassull, V. (2015). Habitar en tierras secas: La tierra cruda como vehículo de habitabilidad en el territorio no irrigado del norte de la Provincia de Mendoza, Argentina. Hábitat Sustentable, 5(2), 69-76. http://revistas.ubiobio.cl/index.php/RHS/ article/view/1931

Pardal March, C. (2009) La hoja interior de la fachada ventilada. Análisis, taxonomía y prospectiva [Tesis doctoral, Universidad Politécnica de Cataluña]. Barcelona, España.

Paricio, I., \& Pardal, C. (2014). Añagazas de la fachada ventilada: ipluvial o revestida? Revista Palimpesto 09.

Patania, F., Gagliano, A., Nocera, F., Ferlito, A., \& Galesi, A. (2010). Thermofluid-dynamic analysis of ventilated facades. Energy and Buildings, 42(7), 1148-1155.

h t tp s://doi.org/10.1016/j. enbuild.2010.02.006
Peci López, F., Jensen, R. L., Heiselberg, P., \& Ruiz de Adana Santiago, M. (2012). Experimental analysis and model validation of an opaque ventilated façade. Building and Environment, 56, 265-275.

https://doi.org/10.1016/j. buildenv.2012.03.017

Peci López, F., \& Ruiz Adana Santiago, M. (2015). Sensitivity study of an opaque ventilated façade in the winter season in different climate zones in Spain. Renewable Energy, 75, 524-533.

https://doi.org/10.1016/j.renene.2014.10.031

Ponte, R. (2008) Mendoza. Aquella ciudad de barro (1a ed.). Consejo Nacional de Investigaciones Científicas Técnicas (CONICET).

Salas Serrano, J. (2000). La industrialización posible de la vivienda latinoamericana. Escala.

Sanjuán, C., Suárez, M., J., González, M., Pistono, J., \& Blanco, E. (2011). Energy performance of an open-joint ventilated façade compared with a conventional sealed cavity façade. Solar Energy, 85(9), 1851-1863. h t t p s : // d o i.org/10.1016/j . solener.2011.04.028

Stazi, F., Tomassoni, F., Veglio, A., \& Di Perna, C. (2011). Experimental evaluation of ventilated walls with an external clay cladding. Renewable Energy, 36, 3373-3385. https://doi.org/10.1016/j.renene.2011.05.016

Suárez, C., \& Molina, J. L. (2015). Análisis del efecto chimenea en fachadas ventiladas opacas mediante correlaciones del flujo másico inducido. Aplicación para el dimensionado de anchos de cámara. Revista Informes de la Construcción, 67, 1-9. http://dx.doi.org/10.3989/ic.13.155

Unidiversidad. (2019). Mendoza ¿está abierta a las nuevas tecnologías en construcción? http://www.unidiversidad.com.ar/hastaque-punto-mendoza-esta-abierta-a-lasnuevas-tecnologias-en-construccion

Vega, G. (2017). El concepto de dispositivo en M. Foucault. Su relación con la "microfísica" y el tratamiento de la multiplicidad. Revista Nuevo Itinerario, 136-158. https://revistas.unne.edu.ar/index.php/nit/ article/view/2038 

(1) Portada: Las bovedadas de Bramante.

Fotografía: Luis Alberto Martínez Camacho (2021) CC BY-NC

\section{(ब) (1) (\$)}

(A) Orientación editorial

\section{Enfoque y alcance}

La Revista de Arquitectura (Bogotá) ( (ISSN 1657-0308 Impresa y E-ISSN 2357-626X en línea) es una publicación científica seriada de acceso abierto, arbitrada mediante revisión por pares (doble ciego) e indexada, en donde se publican resultados de investigación originales e inéditos.

Está dirigida a la comunidad académica y profesional de las áreas afines a la disciplina. Es editada por la Facultad de Diseño y el Centro de Investigaciones (CIFAR) de la Universidad Católica de Colombia en Bogotá (Colombia).

La principal área científica a la que se adscribe la Revista de Arquitectura (Bogotá) según la OCDE es:

Gran área: 6. Humanidades

Área: 6.D. Arte

Disciplina: 6D07. Arquitectura y Urbanismo

También se publican artículos de las disciplinas como 2A02, Ingeniería arquitectónica; 5C03, Estudios urbanos (planificación y desarrollo); 6D07, Diseño.

Los objetivos de la Revista de Arquitectura (Bogotá) son:

- Promover la divulgación y difusión del conocimiento generado a nivel local, nacional e internacional

- Conformar un espacio para la construcción de comunidades académicas y la discusión en torno a las secciones definidas.

- Fomentar la diversidad institucional y geográfica de los autores que participan en la publicación.

- Potenciar la discusión de experiencias e intercambios científicos entre investigadores y profesionales.

- Contribuir a la visión integral de la arquitectura, por medio de la concurrencia y articulación de las secciones mediante la publicación de artículos de calidad.

- Publicar artículos originales e inéditos que han pasado por revisión de pares, para asegurar que se cumplen las normas éticas, de calidad, validez científica, editorial e investigativa.

- Fomentar la divulgación de las investigaciones y actividades desarrolladas en la Universidad Católica de Colombia.
Palabras clave de la Revista de Arquitectura (Bogotá): arquitectura, diseño, educación arquitectónica, proyecto y construcción, urbanismo.

Idiomas de publicación: español, inglés, portugués y francés. Título abreviado: Rev. Arquit.

Titulo corto: RevArq

\section{Políticas de sección}

La revista se estructura en tres secciones correspondientes a las líneas de investigación activas y aprobadas por la institución, y dos complementarias, que presentan dinámicas propias de la Facultad de Diseño y las publicaciones relacionadas con la disciplina.

Cultura y espacio urbano. En esta sección se publican los artículos que se refieren a fenómenos sociales en relación con el espacio urbano, atendiendo aspectos de la historia, el patrimonio cultural y físico, y la estructura formal de las ciudades y el territorio.

Proyecto arquitectónico y urbano. En esta sección se presentan artículos sobre el concepto de proyecto, entendido como elemento que define y orienta las condiciones proyectuales que devienen en los hechos arquitectónicos o urbanos, y la forma como estos se convierten en un proceso de investigación y nuevo de conocimiento. También se presentan proyectos que sean resultados de investigación, los cuales se validan por medio de la ejecución y transformación en obra construida del proceso investigativo. También se contempla la publicación de investigaciones relacionadas con la pedagogía y didáctica de la arquitectura, el urbanismo y el diseño.

Tecnología, medioambiente y sostenibilidad. En esta sección se presentan artículos acerca de sistemas estructurales, materiales y procesos constructivos, medioambiente y gestión, relacionados con los entornos social-cultural, ecológico y económico.

Desde la Facultad. En esta sección se publican artículos generados en la Facultad de Diseño, relacionados con las actividades de docencia, extensión, formación en investigación o internacionalización, las cuales son reflejo de la dinámica y de las actividades realizadas por docentes, estudiantes y egresados; esta sección no puede superar el $20 \%$ del contenido.

Textos. En esta sección se publican reseñas, traducciones y memorias de eventos relacionados con las publicaciones en Arquitectura y Urbanismo.
A Frecuencia de publicación

Desde 1999 y hasta el 2015, la Revista de Arquitectura (Bogotá) publicó un volumen al año, a partir del 2016 se publicarán dos números por año en periodo anticipado, enero-junio y julio-diciembre, pero también maneja la publicación anticipada en línea de los artículos aceptados (versión Post-print del autor).

La Revista de Arquitectura (Bogotá) se divulga mediante versiones digitales (PDF, HTML, EPUB, XML) e impresascon un tiraje de 700 ejemplares, los tiempos de producción de estas versiones dependerán de los cronogramas establecidos por la editorial.

Los tiempos de recepción-revisión-aceptación pueden tardar entre seis y doce meses dependiendo del flujo editorial de cada sección y del proceso de revisión y edición adelantado.

Con el usuario y contraseña asignados, los autores pueden ingresar a la plataforma de gestión editorial y verificar el estado de revisión, edición o publicación del artículo.
A Canje

La Revista de Arquitectura (Bogotá) está interesada en establecer canje con publicaciones académicas, profesionales o científicas del área de Arquitectura y Urbanismo, como medio de reconocimiento y discusión de la producción científica en el campo de acción de la publicación.

Mecanismo

Para establecer canje por favor descargar, diligenciar y enviar el formato: RevArq FP20 Canjes

Universidad Católica de Colombia (2021,
junio-diciembre). Revista de
Arquitectura (Bogotá), 23(2),
I-132. Doi: 10.14718
ISSN: I657-0308
E-ISSN: 2357-626X
Especificaciones:
Formato: $34 \times 24 \mathrm{~cm}$
Papel: Mate II5 g
Tintas: Negro y policromía

A Contacto

Dirección postal:

Avenida Caracas N ${ }^{0} \cdot 46-72$ Universidad Católica de Colombia Bogotá D. C., Colombia Código postal: 111311

Facultad de Diseño Centro de Investigaciones (CIFAR) Sede El Claustro. Bloque "L", 4 piso Diag. 46A N ${ }^{0 .} 15 b-10$ Editor, Arq. César Eligio-Triana

Teléfonos:

+57 (1) $3277300-3277333$

Ext. $3109 ; 3112$ o 5146
Fax: +57 (1) 2858895

Correo electrónico:

revistadearquitectura@ucatolica.edu.co cifar@ucatolica.edu.co

Página WEB:

www.ucatolica.edu.co

Vínculo Revistas científicas

http://publicaciones.ucatolica.edu.co revistas-cientificas

https://revistadearquitectura.ucatolica.edu.co/ 
